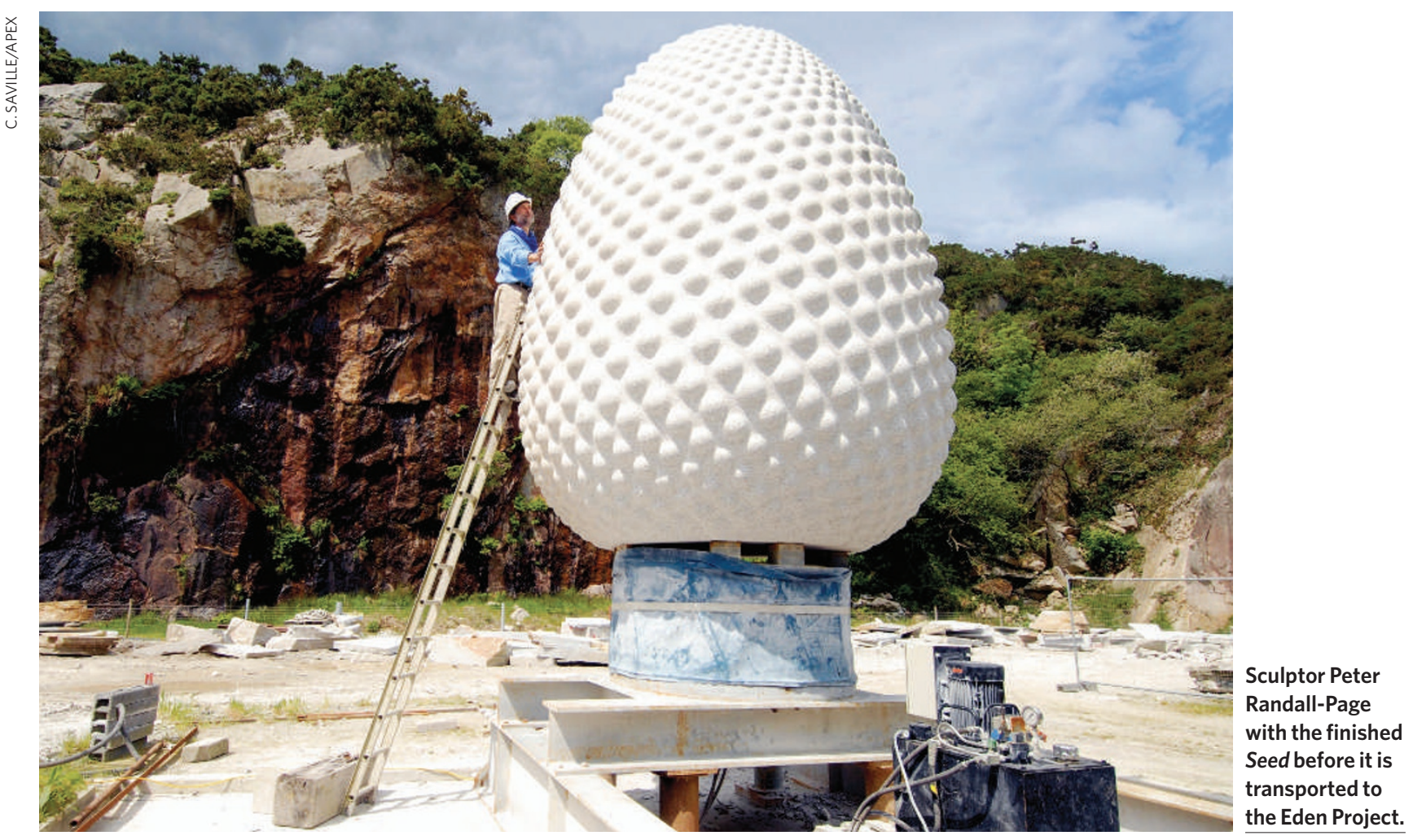

\title{
Consulting nature's pattern-book
}

\section{Peter Randall-Page's massive granite sculpture for the Eden Project emulates natural form.}

Long before scientists applied their analytical skills to underlying regularities of form and process in nature, artists intuitively exploited a repertoire of common patterns that recur across organic and inorganic forms. Some favourites, such as spirals and waves, feature as motifs in an astonishingly wide range of cultures over the ages.

Contemporary artists who are drawn to these patterns begin from similarly intuitive starting points, drawn into the magic of form through our ingrained fascination with order and disorder in natural process. Nowadays the artist, if he or she chooses, can look to a large body of research and writing by scientists who have examined the mechanisms behind appearance.

For their research an artist can turn to distinguished books of popular science such as Philip Ball's The Self-Made Tapestry, John Barrow's The Artful Universe, and the various explorations of the mathematics of complexity by lan Stewart. And, of course, they can always consult the grandfather of geometrical morphologists, D'Arcy Wentworth Thompson, whose 1917 book, On Growth and Form, remains an all-time classic of science writing.

Peter Randall-Page has established himself as one of the foremost explorers of this territory, as a sculptor who has consistently deployed the geometry of natural growth.
He defines his art as founded on "nature's underlying pattern-book of forms".

Seed, the massive granite sculpture recently installed in the education building at the Eden Project, an environmental centre in Cornwall, UK, is a triumphant realization of his vision. Weighing 62 tonnes and standing more than 4 metres tall, its great granite mass is enclosed in the tight embrace of a pod-like chamber at the centre of the building, where it is subject to the infinitely varied caress of natural light.

The curvaceous egg shape of Seed was determined by Randall-Page's sense of the instinctive rightness of its contours in relation to size, mass and material. A set of nodes, systematically graded in their diameter and protrusion, spiral around the form. The relationship to nature is one of principles and resonances, rather than imitation. We think of a pine cone or the arrangement of bud primordia on a shoot.

Randall-Page explains how this phyllotaxis was achieved. "I initially worked with lain Cant, an expert in computer threedimensional modelling. Together we plotted the spiral phyllotaxis pattern onto a virtual three-dimensional form that was then printed out as a three-dimensional rapid prototype model. I had hoped that we would be able to project the virtual image onto the stone itself in order to draw the pattern, but in the event this method proved not to be sufficiently accurate, although it was useful for establishing the arrangement of circles on the very top of the sculpture.

"Eventually, I plotted the pattern of around 1,800 nodes directly onto the surface of the stone using a ruler and compass. I drew two primary spirals traversing the form in opposite directions to represent the two dominant alignments of circles. Using horizontal bands, I was then able to divide each circumference into numerical divisions of two consecutive Fibonacci numbers, in this case 21 and 34. Joining these points created two families of opposing spirals whose intersection represented the centre of each node."

The resulting sculpture is more than simply an adornment to the building that houses it. Randall-Page played an important role in how architect Jolyon Brewis at Grimshaw determined the spiral geometries of the 'sunflower seed' roof that embraces the lofty peak of Seed. The sculpture and building share their natural engineering. They sit in the Eden clay pit as beautiful products of human creativity - what Leonardo da Vinci called "a second nature in the world". Martin Kemp is professor of the history of art at the University of Oxford, Oxford OX11PT, UK. His new book, Seen | Unseen, is published by Oxford University Press. 